\title{
CARACTERIZAÇÃO DE AGRAVOS CAUSADOS POR CÃES E GATOS A SERES HUMANOS NO MUNICÍPIO DE JABOTICABAL, SÃO PAULO, DURANTE O PERÍODO DE 2000 A 2009
}

\author{
Danila Fernanda Rodrigues Frias ${ }^{1}$, Juliana Olivência Ramalho Nunes ${ }^{1}$, Adolorata \\ Aparecida Bianco Carvalho ${ }^{1}$
}

1 UNESP-Jaboticabal

Correspondência: Danila Frias: danilafrias@yahoo.com.br

\begin{abstract}
RESUMO: A falta de controle adequado das populações de cães e gatos, aliada à irresponsabilidade dos seus donos, são fatores que favorecem as agressões por esses animais, gerando um grave problema de saúde publica. O objetivo deste estudo foi caracterizar os agravos causados por cães e gatos do Município de Jaboticabal/SP, no período de 2000 a 2009, visando demonstrar a importância do controle das agressões para redução do risco de transmissão da raiva e do número de profilaxias pós-exposição. Trata-se de um estudo descritivo retrospectivo onde foram analisadas 3.972 das 4.149 fichas de atendimentos antirrábicos humanos no período. A espécie que mais provocou agravos foi a canina. A condição clínica "sadia" do animal no momento da agressão foi declarada em $81,8 \%$ das fichas; em $81,1 \%$ dos casos essa condição se manteve durante todo o período de observação. A maioria dos agravos foram mordeduras, nas mãos e/ou nos pés, com ferimentos múltiplos e superficiais. O número de agravos foi muito elevado, por isso é importante que estratégias para redução sejam implementadas por meio de ações de promoção à saúde, atividades educativas e fortalecimento dos serviços integrados de médicos e veterinários, para que a decisão de se instituir a profilaxia antirrábica pós-exposição seja tomada com critério e segurança.
\end{abstract}

Palavras-chave: cães e gatos; mordedura; profilaxia pós-exposição; raiva

\section{CHARACTERIZATION OF INJURIES CAUSED BY DOGS AND CATS TO HUMANS IN THE MUNICIPALITY OF JABOTICABAL, SÃO PAULO, BETWEEN THE YEARS 2000 AND 2009}

\begin{abstract}
The lack of proper control of dogs and cats population and irresponsible pet ownership are factors that favor animal attack and pose serious public health concern. The aim of the present study was to characterize the injuries caused by dogs and cats to humans in the municipality of Jaboticabal, between the years 2000 and 2009, with a view to demonstrate the importance of aggression control in reducing both the risk for rabies transmission and the number of post exposure prophylaxis administration. A retrospective and descriptive study was conducted. 3,972 out of 4,149 records of patients who sought the public health service after being attacked by an animal were analyzed. Dogs were the species that most caused injuries. In the moment of the attack, the dog's clinical status was declared as "healthy" in $81.8 \%$ of records; and in $81.1 \%$ of cases this status were maintained throughout the entire observation period of the aggressor. Most of injuries were characterized by bites in hands or feet, with multiple and superficial wounds. The number of injuries was high, therefore it is crucial to implement strategies to reduce injuries caused by animal attack through health promotion, educational activities and strengthening of integration of public health and veterinary services so that the decision to institute post-exposure prophylaxis is taken with criteria and safety.
\end{abstract}

Key Words: cats and dogs; bites; post-exposure prophylaxis; rabies 

Jaboticabal, São Paulo, durante o período de 2000 a 2009

\section{INTRODUÇÃO}

Apesar do convívio quase sempre pacífico entre os seres humanos e o cão, a agressividade canina manifestase contra as pessoas muitas vezes influenciada por hábitos culturais ou pelo desconhecimento das bases sociológicas de convivência entre espécies, provocando distúrbios de comportamento (Jenkins et al., 2002). Geralmente essas alterações comportamentais acontecem em situações de defesa de território, dos filhotes, do alimento, por medo, por dominância, pela posse de objetos, por condicionamento ou adestramento, ou até mesmo por brincadeira (São Paulo, 2011).

A mordedura causada por cão é motivo de grande preocupação devido ao favorecimento de ocorrência de infecções por alguns agentes etiológicos da cavidade bucal, e também por existir a possibilidade de transmissão de zoonoses, especialmente a raiva, constituindo assim um grave problema para a comunidade, para outros animais e para a saúde pública (Dietz, 2000; Jenkins et al., 2002). Além disso, as agressões também causam traumas psíquicos e físicos (ferimentos leves ou graves, mutilações ou óbito) (São Paulo, 2011).

Em crianças, a mordedura de cão destaca-se pela gravidade das lesões, muitas vezes com sequelas, tanto emocionais, devido ao estresse vivido pela criança, como físicas, decorrentes das cicatrizes e desfigurações (Filócomo et al., 2002; Ballesteros et al., 2003).

Os dados epidemiológicos envolvendo agravos com cães no Brasil ainda são escassos, porém, órgãos do Ministério da Saúde destacam que a subnotificação é muito alta, devido a grande ocorrência de lesões leves desprezadas pelas pessoas envolvidas (Baracat et al., 2000; Alonso \& Prada, 2005).
O crescimento e o adensamento populacional urbano contribuem para uma estreita convivência dos seres humanos com os animais, o que facilita a ocorrência de agressões e, consequentemente, a transmissão de zoonoses. Dentre as agressões, as mais comuns são a mordedura e a arranhadura, levando a uma maior preocupação com relação ao risco da raiva (Dietz, 2000).

A raiva ocorre de maneira endêmica em diversos países do mundo, inclusive no Brasil (Velasco-Villa et al., 2006). Em áreas urbanas, onde as medidas de controle não atingem seu objetivo, a espécie de maior relevância epidemiológica para a transmissão do vírus é o cão, seguido pelo morcego não-hematófago (De Mattos et al., 2001).

No Brasil, a redução na incidência da raiva canina foi de $91 \%$, entre os anos de 1990 e 2006; em 2009, ocorreram 26 casos. A expectativa é que a raiva humana transmitida por cães e gatos seja erradicada do país. Mas, mesmo com todo o controle por meio da vacinação dos animais domésticos, captura de animais errantes e bloqueio de focos, é preciso buscar uma eficiente vigilância epidemiológica dos casos transmitidos por animais silvestres para evitar novos surtos em cães (Brasil, 2009; Brasil, 2011).

Devido à influência de fatores que levam cães a apresentarem alterações de comportamento, 0 número de pessoas agredidas por essa espécie no Brasil ainda é muito alto, com índice de tratamento pós-exposição contra raiva também muito elevado (Schneider et al., 2007). Por isso, o presente trabalho teve como objetivo caracterizar os agravos causados por cães e gatos na população do Município de Jaboticabal, São Paulo, no período de 2000 a 2009, visando prevenir a ocorrência de agressões e demonstrar a importância do seu controle para consequente 
redução do risco de transmissão da raiva e do número de tratamentos pósexposição.

\section{MATERIAL E MÉTODOS}

\section{Estudo descritivo}

Após autorização do secretário de saúde do Município de Jaboticabal, a pesquisa foi realizada por meio de um levantamento de dados registrados nas fichas de investigação de atendimento antirrábico humano, arquivadas no Centro de Saúde "Albertino Affonso". 1.1. Obtenção e digitalização dos dados

As fichas de investigação de atendimento antirrábico humano estavam arquivadas na pasta (prontuário) de cada paciente. Para facilitar seu resgate, a Secretaria de Vigilância Epidemiológica do Município de Jaboticabal disponibilizou uma lista do Sistema de Informações de Agravos de Notificação (SINAN) contendo os nomes das pessoas envolvidas em acidentes com animais e que procuraram atendimento durante 0 período de estudo. A partir desta, efetuou-se uma pesquisa no computador do Centro de Saúde que gerou os números dos prontuários dessas pessoas. A ficha de investigação de atendimento anti-rábico era extraída da pasta de cada um dos pacientes e, após imediata digitalização dos dados, devolvida ao arquivo.

\subsection{Formação do banco de dados}

Foram analisadas 4.149 fichas, relativas ao período de $1^{\circ}$ de janeiro de 2000 a 31 de dezembro de 2009, e foram utilizadas neste estudo 3.972 fichas que foram as relativas a acidentes causados por cães e gatos.

A ficha é composta por 64 campos distribuídos em seis sessões diferentes.

Foram utilizados para a presente investigação os seguintes campos: espécie do animal agressor e condição vacinal do mesmo; condição do animal no ato do acidente e durante o período de observação; tipo de exposição; região anatômica atingida; características do ferimento, quanto à extensão e número de lesões, e quanto à profundidade.

\subsection{Tabulação dos dados}

Os dados foram tabulados em planilhas do software Microsoft Office Excel 2010.

\section{RESULTADOS E DISCUSSÃO}

\section{Caracterização do animal envolvido no agravo}

\subsection{Espécie}

Durante os anos de estudo, $85,5 \%$ dos agravos foram provocados por cães, 10,2\%, por gatos e 4,3\% por outras espécies, sendo, portanto, o cão responsável pela maioria dos acidentes, em todos os anos. Outras investigações também detectaram a espécie canina como a causadora da maioria dos agravos em seres humanos (Pinto \& Alleoni, 1986; Carvalho et al., 2002; Rigo \& Honer, 2002; Ostrowska et al., 2003; Hossain et al., 2004; Kilic et al., 2006; Morais et al., 2006; Moreno et al., 2006; Poerner \& Pereira, 2006; Rolim et al., 2006; Sengoz et al., 2006; Tomasiewicz et al., 2006). Este fato pode ser compreendido quando se constata que o aumento da população canina está diretamente relacionado com o aumento da população humana (Garcia et al., 1999). O relacionamento promíscuo que o ser humano tem com os animais de estimação, ligado aos fatores de dominância e submissão dos cães e do seu dono, favorece o risco de agressões.

No Gráfico 1 nota-se que no ano de 2001 ocorreu um pico de agravos por cães, com visível queda em 2002, mantendo-se mais ou menos constante até o final dos anos de estudo, porém, com tendência a uma ligeira queda. Já o 

Jaboticabal, São Paulo, durante o período de 2000 a 2009

número de acidentes com gatos foi praticamente constante.

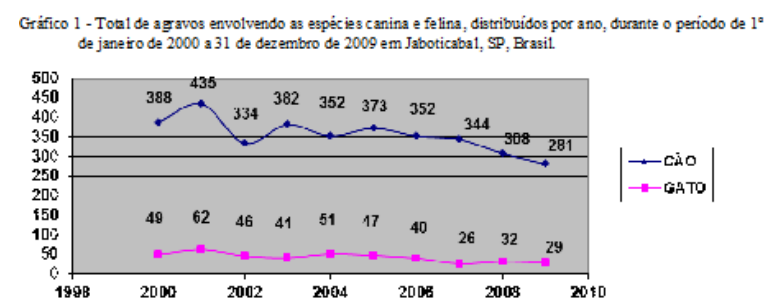

\subsection{Condição clínica do animal no momento do agravo e durante o período de observação}

A observação do animal no momento da agressão e durante os dez dias preconizados são de fundamental importância para o emprego de uma profilaxia adequada. No presente estudo, em $81,8 \%$ dos casos o animal foi declarado sadio no momento do agravo e $81,1 \%$ mantiveram-se sadios durante o período de observação; mas muitas fichas tinham este campo não preenchido. Resultados semelhantes foram encontrados por outros autores, ou seja, a maioria dos animais estavam sadios, tanto no momento da agressão quanto durante 0 período de observação, e muitas fichas estavam incompletas (Ribeiro Netto \& Machado, 1970; Pinto \& Alleoni, 1986; Garcia et al., 1999; Carvalho et al., 2002; Rigo \& Honer, 2002; Poerner \& Pereira, 2006; Rolim et al., 2006).

A informação "sadio" para condição clínica do animal no momento da agressão e durante o período de observação, é um importante subsídio para potencial dispensa de profilaxia antirrabica humana.

\section{Caracterização dos agravos}

A caracterização do agravo também é um fator a ser analisado na instituição da profilaxia. Com relação às características do ferimento, os acidentes devem ser avaliados quanto ao local ou região anatômica atingida (mucosa, cabeça, pescoço, mãos, pés, tronco, membros superiores e membros inferiores); quanto à profundidade (superficiais - sem presença de sangramento, e profundos - com sangramento); e quanto à extensão e número de lesões (única - apenas uma porta de entrada para o vírus, e múltipla - várias portas de entrada) (Brasil, 2002; Associação Médica Brasileira, 2011).

Associando-se a condição clínica do animal no momento do agravo com a espécie do animal agressor, pode-se classificar o acidente como grave ou leve e, assim, orientar a correta profilaxia (São Paulo, 2000). Os leves referem-se aos acidentes superficiais, no tronco e membros, exceto mãos e pés, decorrentes de mordedura ou arranhadura superficiais ou lambedura de lesões superficiais. Já os graves são decorrentes de acidentes com qualquer tipo de ferimento ou lambedura de lesões, em mucosas, cabeça, pescoço, mãos e pés; ferimentos extensos ou múltiplos e profundos, mesmo que puntiformes, em qualquer parte do corpo; lambedura de mucosas, mesmo que intactas; e qualquer acidente com morcego (São Paulo, 2000; Brasil, 2002; Associação Médica Brasileira, 2011).

$\mathrm{Na}$ Tabela 1 estão descritos os acidentes ocorridos em Jaboticabal durante 0 período estudado. A mordedura representou $84,4 \%$ dos agravos, sendo mãos e pés as regiões anatômicas mais atingidas (33\%). Com relação ao tipo de ferimento, o múltiplo representou $69,6 \%$ dos agravos, e o superficial, $56,2 \%$.

$\mathrm{Em}$ outras pesquisas a mordedura também teve grande destaque, sendo sempre o agravo de maior ocorrência (Pinto \& Alleoni, 1986; Garcia et al., 1999; Del Ciampo et al., 2000; Carvalho et al., 2002; Rigo \& Honer, 2002; Poerner \& Preira, 2006; Rolim et al., 2006). Mas, tanto em países desenvolvidos como em subdesenvolvidos, os registros obtidos são escassos e não mostram a 
verdadeira dimensão do problema (Weiss et al., 1998; Osverall \& Love, 2001).

Outra justificativa para que mordedura seja a mais frequente ocorrência é o fato do indivíduo se preocupar em relatar esse tipo de acidente devido à gravidade da lesão e á possibilidade de estar relacionada com a raiva, o que não acontece quando ocorre apenas uma arranhadura ou lambedura.

Quanto ao tipo de ferimento, destacou-se o ferimento único em alguns trabalhos (Carvalho et al., 2002; Poerner \& Pereira, 2006). Mas houve também a ocorrência em maior escala de ferimentos múltiplos, como foi citado em um trabalho realizado em Campo Grande/MS, com ferimentos múltiplos em $48,6 \%$ dos acidentes, resultado este semelhante ao encontrado em Jaboticabal (Rigo \& Honer, 2002).

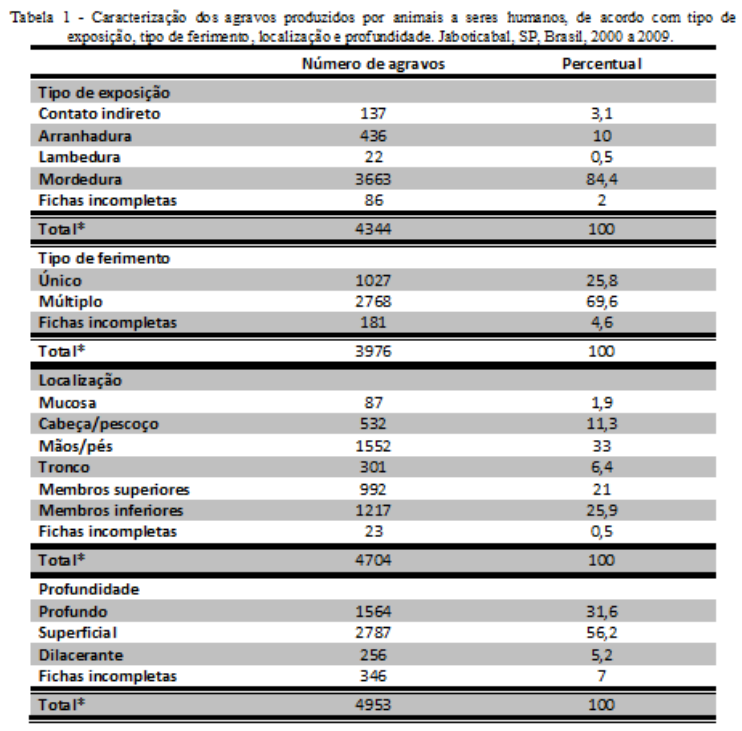

Quando se avalia a região
anatômica mais atingida, existe divergência entre trabalhos. Alguns relatam os membros inferiores (Carvalho et al., 2002; Rigo \& Honer, 2002; Rolim et al., 2006), outros se referem aos membros superiores (Garcia et al., 1999), e também citam o local mais atingido as mãos e os pés, sendo este último igual ao dado encontrado neste trabalho (Ostrowska et al., 2003; Hossain et al., 2004; Poerner \& Pereira, 2006).

O grau de profundidade do ferimento mais encontrado na presente pesquisa foi superficial, assim como por outros autores (Carvalho et al., 2002; Rigo \& Honer, 2002; Poerner \& Pereira, 2006; Sengoz et al., 2006).

Em Jaboticabal, no período de estudo, a maioria dos agravos foram classificados como graves, uma vez que se caracterizavam por mordeduras nas mãos e com ferimentos múltiplos, o que levaria a indicação de profilaxia pósexposição. Porém, para a instituição de profilaxia pós-exposição, ainda deve ser considerado: o estado de saúde do animal no momento da agressão, ou seja, avaliar se o animal estava sadio ou apresentava sinais sugestivos de raiva; as circunstâncias em que o acidente ocorreu - se foi provocado ou não. Ademais, também deve-se sempre lembrar que 0 animal pode agredir devido à sua índole ou adestramento inadequado (São Paulo, 2000; Brasil, 2002 ; Associação Médica Brasileira, 2011).

Também deve ser feita a observação do animal envolvido no agravo, pois a excreção do vírus pela saliva só ocorre após o final do período de incubação, entre quatro e dois dias antes do início dos sintomas, e perdura até a morte do animal, que sobrevive de três a cinco dias após o início do quadro clínico; por este motivo, cães e gatos aparentemente sadios devem ser observados durante 10 dias, a partir da data do acidente (São Paulo, 2000).

Também devem ser avaliados os hábitos de vida e condição sanitária do animal, ou seja, se são domiciliados (animais que têm proprietário e saem à rua contidos, recebem vacinas e são submetidos a controles clínicos periódicos); semi-domiciliados (animais que têm proprietário, mas ficam fora do domicílio desacompanhados, por 

Jaboticabal, São Paulo, durante o período de 2000 a 2009

períodos indeterminados, recebem vacinas e algum cuidado); comunitários ou de vizinhança (não têm dono, ficam soltos nas ruas, mas as pessoas os alimentam); errantes (são independentes, vivem soltos, não recebem nenhuma atenção, alimentamse de restos descartados, abrigam-se em locais públicos e competem com outros animais pela sobrevivência) (São Paulo, 2000). Avalia-se, ainda a área geográfica de procedência do animal, se é considerada área de raiva controlada ou não (São Paulo, 2000; Brasil, 2002; Associação Médica Brasileira, 2011).

Todos esses fatores devem ser analisados de forma muito criteriosa para que não sejam instituídos tratamentos desnecessários que, além de risco à saúde, podem gerar desperdícios aos cofres públicos. No Brasil, em 2005, o Ministério da Saúde gastou com as ações de vigilância epidemiológica para raiva cerca de $R \$$ 66,4 milhões, sendo grande parte desses recursos destinados à aquisição de imunobiológicos para profilaxia pósexposição em humanos (Brasil, 2011).

\section{CONCLUSÃO}

O número de agravos a pessoas envolvendo cães e gatos, no período estudado, foi muito elevado. Isso demonstra a necessidade de se estabelecer estratégias efetivas para a redução de agressões, por meio de ações de promoção à saúde, envolvendo autoridades competentes. Atividades educativas em escolas e para a população em geral sobre controle populacional de animais, posse responsável e comportamento animal, devem ser instituídas para diminuir o número de agravos. $\mathrm{O}$ fortalecimento da integração dos serviços médicos e veterinários no atendimento às pessoas envolvidas em agravos com animais deve ocorrer para que haja uma adequada avaliação do tipo e das circunstâncias do agravo, das condições do animal agressor e do risco epidemiológico da doença no município, permitindo, assim, que a decisão de se instituir ou não profilaxia antirrábica pósexposição seja tomada com critério e segurança.

\section{REFERÊNCIAS}

ALONSO, M.; PRADA, B. Estudo de caso de agressões por cães no município de Araraquara, São Paulo, Brasil. 2005. [Monografia]. Faculdade de Ciências Farmacêuticas, Araraquara, São Paulo, 2005.

ASSOCIAÇÃO MÉDICA BRASILEIRA. CONSELHO FEDERAL DE MEDICINA. Sociedade Brasileira de Pediatria. Projeto Diretrizes: Vacina Contra - Raiva Humana. Disponível em: http://www.projetodiretrizes.org.br/projeto_diretri zes/120.pdf. Acesso em: 21 set 2011

BALLESTEROS, M. et al. Differential ranking of causes of fatal versus non-fatal injuries among US children. Injury Prevention, v.9, p.176-187, 2003.

BARACAT, E. C. E. et al. Acidentes com crianças e sua evolução na região de Campinas, SP. Jornal de Pediatria, v.76, n.5, p.368-374, 2000.

BRASIL. Ministério da Saúde. Mapas da raiva no Brasil, 2009. Disponível em:

http://portal.saude.gov.br/portal/arquivos/pdf/ma pas_raiva_2009_dados_finais.pdf. Acesso em: 21 mar 2011.

BRASIL. Ministério da Saúde. Normas técnicas de tratamento profilático anti-rábico

humano. Disponível em:

http://www.saude.rj.gov.br/Acoes/NORMA_TEC NICA_DA_RAIVA_2002.pdf. Acesso em 10 jun 2011.

BRASIL. Ministério da Saúde. Brasil atento a

Raiva Humana. Disponível em:

http://portal.saude.gov.br/portal/saude/visualizar texto.cfm?idtxt=25213. Acesso em: 08 maio 2011.

CARVALHO, W. O.; SOARES, D. F. P. P.; FRANCESCHI, V. C. S. Características do atendimento prestado pelo serviço de profilaxia da raiva humana na rede municipal de saúde de 
Maringá-Paraná, no ano de 1997. Informe Epidemiológico do SUS, v.11, n.1, p.25-35, 2002.

DEL CIAMPO, L. A. et al. Acidentes com animais domésticos na infância e adolescência. Pediatria, São Paulo, v. 22, n. 4, p. 319-324, 2000.

DE MATTOS, C. A.; DE MATTOS, C. C.; RUPPRECHT, C. E. Rhabdoviruses. In: KNIPE, D. M.; HOWLEY, P. M. Fields Virology. Philadelphia: Lippincott Williams \& Wilkins, 2001. p. $1245-1278$.

DIETZ, G. Perfil epidemiológico dos pacientes agredidos por animais no município de Pirassununga/SP, entre os anos de 1997 a 1999. 2000. [Monografia].Faculdade de Ciências Farmacêuticas, Araraquara, São Paulo, 2000.

FILOCOMO, F. R. F. et al. Study of accidents involving children assited at an emergency ward. Revista Latino-Americana de Enfermagem, v.10, n.1, p.41-47, 2002.

GARCIA, R. C. M. et al. Análise de tratamento anti-rábico humano pós-exposição em região da Grande São Paulo, Brasil. Revista de Saúde Pública, v.33, n.3, p.295-301, 1999.

HOSSAIN, J. et al. Audit of rabies post-exposure prophylaxis in England and Wales in 1990 and 2000. Communicable Disease and Public Health, v.7, n.2, p.105-111, 2004.

JENKINS, S. R. et al. Compendium of animal rabies prevention and control. Journal of American Veterinary Medical Association, v.221, n.1, p.44-48, 2002.

KILIC, B. et al. An important public health problem: rabies suspected bites and posexposure prophylaxis in a health district in Turkey. International Journal of Infectious Diseases, v.10, n.3, p.248-254, 2006.

MORAIS, N. B.et al. The prophylaxis profile of the human rabies in the state of Ceará. In: Reunião Internacional de Raiva nas Américas, XVII., 2006, Brasilia. Anais... Brasília: Ministério da Saúde, 2006. p.169.

MORENO, J. O.; MORAIS, N. B.; VASCONCELOS, D. C. The profile of the prophylaxis of human rabies in the regional cell of health of Caucaia - CE. In: Reunião
Internacional de Raiva nas Américas, XVII., 2006, Brasilia. Anais... Brasília: Ministério da Saúde, 2006. p.172.

OSTROWSKA, J. D. et al.Evaluation of the prophylactic anti-rabies vaccination program for adults carried out by the Center for Rabies Prevention in Bialystok, 1992-2001. Medycyna Pracy, v. 54, n. 5, p. 53- 456, 2003.

OVERALL, K. L.; LOVE, M. Dog bites to humans - demography, epidemiology, injury, and risk. Journal of the American Veterinary Medical Association, v.218, n.12, p.1923-1934, 2001.

PINTO, C. L.; ALLEONI, E. S. Aspectos da vigilância epidemiológica da raiva em subregiões administrativas do Estado de São Paulo, Brasil, 1982-1983. Revista de Saúde Pública, v.20, n.4, p.288-292, 1986.

POERNER, A. L.; PEREIRA, M. J. S.

Epidemiology of human rabies post-exposure prophylaxis and relationship between rabies control strategies in Centro Sul area of Rio de Janeiro, Brazil. In: Reunião Internacional de Raiva nas Américas, XVII., 2006, Brasilia. Anais... Brasília: Ministério da Saúde, 2006. p. 170.

RIBEIRO NETTO, A.; MACHADO, C. G. Alguns aspectos epidemiológicos da exposição humana, ao risco de infecção pelo vírus da raiva na cidade de São Paulo, Brasil. Revista do Instituto de Medicina Tropical de São Paulo, v.12, p.16-30, 1970

RIGO, L.; HONER, M. R. Análise da profilaxia da raiva humana em Campo Grande, MS, Brasil em 2002. Cadernos de Saúde Pública, v.21, n.6, p.1939-1945, 2005.

ROLIM, R. L. P.; LOPES, F. M. R.; NAVARRO, I. T. Aspectos da vigilância epidemiológica da raiva no Município de Jacarezinho, Paraná, Brasil, 2003. Seminário: Ciências Agrárias, v.27, n.2, p.271-280, 2006.

SÃO PAULO. Secretaria de Estado da Saúde. Instituto Pasteur. Profilaxia da raiva humana. 2 ed. São Paulo: Instituto Pasteur, 2000. n.4, 33p.

SÃO PAULO. Secretaria de Estado da Saúde. Instituto Pasteur. Por que ocorrem agressões? Disponível em:

http://www.pasteur.saude.sp.gov.br/cao/cao_02. htm. Acesso em 18 jun 2011. 

Jaboticabal, São Paulo, durante o período de 2000 a 2009

SCHNEIDER, M. C. et al. Current status of human rabies transmitted by dogs in Latin America. Cadernos de Saúde Pública, v.23, n.9, p. 2049-2063, 2007.

SENGOZ, G.et al. Evaluation of cases admitted to a Center in Istanbul, Turkey in 2003 for rabies vaccination and three rabies cases followed up in the last 15 years. Japanese Journal of Infectious Diseases, v.59, n.4, p 254-257, 2006.

TOMASIEWICZ, K. et al. Post-exposure antirabies prophylaxis in Lublin province (Eastern Poland) in 2004-2005. Annals of Agricultural and Environmental Medicine, v.13, n.2, p.337340, 2006.

VELASCO-VILLA, A. et al. Molecular diversity of rabies viroses associated with bats in México and other countries of the Américas. Journal of Clinical Microbiology, v.44, n 5, p.1697-1710, 2006.

WEISS, H. B.; FRIEDMAN, D. I.; COBEN, J. H. Incidence of dog bite injuries treated in emergency departments. Journal of the American Medical Association, v.279, n.1, p.51-53, 1998. 